\title{
Therapeutic Application of High-Frequency rTMS Combined with Intensive Occupational Therapy for Pediatric Stroke Patients with Upper Limb Hemiparesis: A Case Series Study
}

\author{
Masachika Niimi, Wataru Kakuda, Toru Takekawa, Ryo Momosaki, Takatoshi Hara, \\ Hiroshi Ito, Yumi Kameda, Masahiro Abo \\ ${ }^{1}$ Department of Rehabilitation Medicine, The Jikei University School of Medicine, Tokyo, Japan \\ Email: motoquiso@gmail.com
}

Received March 8, 2013; revised April 11, 2013; accepted May 12, 2013

Copyright (C 2013 Masachika Niimi et al. This is an open access article distributed under the Creative Commons Attribution License, which permits unrestricted use, distribution, and reproduction in any medium, provided the original work is properly cited.

\begin{abstract}
Low-frequency repetitive transcranial magnetic stimulation (LF-rTMS) applied to the non-lesional hemisphere is reported to significantly improve motor function of the affected upper limb in adult stroke patients with hemiparesis. For pediatric stroke patients, the beneficial effects of LF-rTMS have been already confirmed in a randomized controlled study. However, there is no report of therapeutic application of high-frequency rTMS (HF-rTMS) in this patient population. In this case series study, we introduced HF-rTMS combined with intensive occupational therapy (OT) in two pediatric hemiparetic patients. We studied two children (8- and 9-year-old boys, both right-handed) with post-stroke upper limb hemiparesis (chronic phase). Both patients underwent 22 treatment sessions of HF-rTMS/OT during 15-day hospitalization. The HF-rTMS was applied over the lesional motor cortex at a frequency of $10 \mathrm{~Hz}$ for 15 minutes in each session. One session of intensive OT consisted of 30-min one-to-one training and 30-min self-exercise. Motor function of the affected upper limb was serially evaluated with Fugl-Meyer Assessment (FMA), Wolf Motor Function Test (WMFT), Simple Test for Evaluating hand Function (STEF), and Ten-second Test. Neither of the patients showed any adverse effects. Both patients showed improvement of motor function in the affected upper limb and were able to use the affected upper limb in some activities of daily living. In the two post-stroke pediatric patients, HF-rTMS/OT was safe and improved upper limb muscle function. Confirmation of these effects in a larger population is needed.
\end{abstract}

Keywords: Pediatric Stroke; Repetitive Transcranial Magnetic Stimulation; Occupational Therapy; Upper Limb Hemiparesis; Motor Recovery

\section{Introduction}

Pediatric stroke is not a common disease compared with adult stroke. The annual incidence rate of pediatric stroke is estimated to be $2.4 / 100,000$ in the United States [1]. While the leading cause of adult stroke is arteriosclerosis, the underlying causes of pediatric stroke relate to rare congenital abnormalities manifested as cardiovascular disorders, prothrombotic disorders, arteriopathies and cerebral arterial abnormalities rather than aging [2]. It seems that the diagnosis and treatment of pediatric stroke is often delayed, due to the uncommonness of the condition [3]. It has been reported that hemiparesis is the most frequent symptom found in ischemic stroke in children aged $>1$ year [4,5]. Functional impairment after stroke, including hemiparesis, critically influences the activities of daily living (ADL) over the lifetime. The majority of pediatric patients with post-stroke hemiparesis have disabilities and reduced quality of life (QOL) [6]. Although motor impairment after stroke can be expected to recover more significantly in pediatric than in adult patients due to differences in neuroplasticity, no effective therapy for poststroke hemiparesis in pediatrics is available at present.

In adult stroke, on the other hand, various therapeutic interventions for post-stroke hemiparesis have been proposed, and the efficacy of certain interventions has been examined and already confirmed in well-designed clinical trial [7]. Recently, repetitive transcranial magnetic stimulation (rTMS) has received attention based on its beneficial effect for post-stroke patients. The rTMS is a non- 
invasive technique of stimulation that modulates the brain plasticity and leads to improvement of motor function. The rTMS has different effects on the brain when applied at high- or low-frequency. At our department, the combination of low-frequency rTMS (LF-rTMS) and intensive occupational therapy (OT) has been used in adult poststroke patients. This combined intervention is based on the concept that massive training facilitates the development of beneficial functional reorganization when provided with rTMS to "precondition" the brain. A pilot study of LF-rTMS/OT showed that this combination treatment is safe and can potentially improve motor deficit [8]. So far, however, only a few researchers have introduced rTMS to pediatric patients as a therapeutic tool [9]. Moreover, there are no reports on the use of high-frequency rTMS (HF-rTMS)/OT in pediatric hemiparetic patients. The purpose of this case series study was to evaluate the safety, feasibility and efficacy of HF-rTMS combined with intensive OT in two pediatric stroke patients with upper limb hemiparesis.

\section{Subjects}

Two male pediatric patients with stroke and upper limb hemiparesis were the subjects of this study. The clinical characteristics of the patients are summarized in Table $\mathbf{1 .}$ Both patients had mild upper limb hemiparesis caused by cerebral infarction. The latency between stroke and this study was more than two years. Patient 1 was right-handed with left upper limb hemiparesis, whereas Patient 2 was right-handed with right upper limb hemiparesis. The location of lesion, which was confirmed with non-contrasted brain CT, was right basal ganglia in Patient 1 and left posterior limb of the internal capsule in Patient 2. None had any other neurological symptoms apart from upper limb hemiparesis. Mental development and academic achievement were normal. None had history of epilepsy. Furthermore, none had pathological conditions known to be contraindication for rTMS according to the guidelines of Wassermann [10].

The ethics committee of our university approved the study protocol and informed consent was obtained from the parents before study entry. Prior to the intervention, we explained in detail to the patients and their parents the purpose, method and risk of the intervention. The intervention was applied during 15-day hospitalization at our department as an in-patient treatment. One treatment session consisted of high-frequency rTMS over the lesional hemisphere followed by intensive OT comprising of oneto-one training and self-exercise. Thus, during the hospitalization, 22 treatment sessions were provided for each patient. The patients were frequently interviewed and examined for possible fatigue or adverse effects, throughout the duration of hospitalization. In principle, the parents
Table 1. Clinical characteristics of two studied patients.

\begin{tabular}{|c|c|c|}
\hline & Patient 1 & Patient 2 \\
\hline $\begin{array}{l}\text { Age at admission } \\
\text { (years) }\end{array}$ & 8 & 9 \\
\hline Gender & Male & Male \\
\hline $\begin{array}{l}\text { Time between onset of stroke } \\
\text { and treatment (months) }\end{array}$ & 73 & 26 \\
\hline Type of stroke & Cerebral infarction & Cerebral infarction \\
\hline Location of lesion & Right BG & Left PLIC \\
\hline Severity of paresis & $\begin{array}{l}\text { Mild (BRS for } \\
\text { hand-fingers of 5) }\end{array}$ & $\begin{array}{l}\text { Mild (BRS for } \\
\text { hand-fingers of 5) }\end{array}$ \\
\hline Side of the affected limb & Left & Right \\
\hline Handedness & Right-handed & Right-handed \\
\hline
\end{tabular}

were permitted to visit the patients and observe the rehabilitation program.

\section{Methods}

\subsection{Application of rTMS}

The rTMS was applied using a $70 \mathrm{~mm}$ figure 8 coil and MagPro R30 stimulator (MagVenture Company, Farum, Denmark). The patient was held in the sitting position during the application of rTMS. In one rTMS session, HF-rTMS $(10 \mathrm{~Hz})$ was applied to the lesional hemisphere over the primary motor areas for 15 minutes. In each 15minute session, intermittent $10-\mathrm{Hz}$ rTMS was applied in 10 -sec trains with 50 -sec intervals between trains (total 1500 pulses). The optimal site of stimulation on the scalp was defined as the location where the largest motor evoked potentials (MEPs) in the first dorsal interosseous (FDI) muscle of the affected upper limb was elicited on the surface electromyogram. The motor threshold (MT) of FDI muscle of the affected upper limb was defined as the lowest intensity of stimulation that could activate MEPs of the muscle. The intensity of stimulation was set at $90 \%$ of the measured MT of the FDI muscle.

\subsection{Rehabilitative Program of Intensive OT}

For adult stroke patients treated with rTMS, we developed intensive OT program consisting of 60-min one-toone training and 60-min self-exercise [8]. However, this program seemed unsuitable for pediatric patients because it is monotonous and long for children, and requires continuous concentration. Therefore, we modified the program to 30-min one-to-one training and 30-min self-exercise to suit and be accepted by the pediatric patients. The one-to-one training included shaping and repetitive task practice techniques. During the self-training, the re- 
petitive task practice techniques were mainly performed without supervision by the occupational therapist. The modified training program was pre-pared for the pediatric patients to concentrate on the tasks. For instance, when the patients performed a sanding task in the one-to-one training, they were asked to push a ball off a table with the sanding device. The patients colored a paper on the wall with the paretic hand painted, elevating the paretic upper limb. The patient and the occupational therapist pushed a ball to each other using the sanding device with the hand dorsal flexion, face to face across the table. During self-exercise, the patients made the shapes of animals and foods using clay. The patients performed each task in the presence of their parents. At discharge from the hospital, the occupational therapist offered detailed instructions for daily home self-exercise.

\subsection{Clinical Evaluation}

Fugl-Meyer Assessment (FMA), Wolf Motor Function Test (WMFT), Simple Test for Evaluating hand Function (STEF) and Ten-second Test were used serially to evaluate motor function in the affected upper limb. The FMA is a performance-based quantitative measure that assesses various impairments in stroke patients [11]. The section of upper limb motor function of the FMA consists of 33 items. The maximum motor performance score for the upper limbs is 66 points, as each item is rated on a threepoint ordinal scale $(0=$ cannot perform, $1=$ can perform partially, 2 = can perform fully). The WMFT includes 15 functional timed tasks and the performance time of each task is measured [12]. In this study, the quality of the movement was mainly evaluated, as the function ability score (FAS). In the FAS, the effort, smoothness and overall quality are rated on a 6-point ordinal scale $(0=$ does not attempt, $5=$ normal movement), and the score of each task is summed. The STEF which is broadly used in clinical settings in Japan, consists of 10 subtests designed on the basis of various activities [13]. The examinee is asked to grasp and move objects of different size, shape, weight and texture. The time to complete each subtest is measured and rated on a 10-point ordinal scale. Consequently, the score in each subtest is summed. The Ten-second Test consists of three types of tests [14]. The examinee performs three types of hand-fingers movement (finger individual movement, hand pronation and supination and finger tapping) as quickly as possible for 10 seconds, and the number of movements is counted. These evaluations were serially performed at admission, at discharge and 4 weeks after discharge. In addition, change in ADL functions and QOL were estimated though frequent interviews with the patients and their parents and the observation of the patients' daily life in the ward by the physicians, nurses and occupational therapists at our department.

\section{Results}

During 15-day hospitalization, neither of the patients showed any adverse effect. Both patients completed the protocol. Changes in the measures applied for the study are summarized in Table 2. The score of STEF and the sum of FAS on WMFT increased in both patients. The number of movements in Ten-Second Test also increased in both of them at 4 weeks after discharge. Notably, the FMA score in Patient 2 showed 3-point increase during the period between the discharge and 4 weeks after discharge. Changes in ADL function using the affected upper limb are summarized in Table 3 . At discharge, Patient 1 was able to pass the affected arm through the sleeve of a shirt and fasten the buttons on a shirt smoothly. He was also able to grasp a bowl stably, and move the bowl to his mouth without any difficulty. At 4 weeks post-discharge, the general ADL functions remained in good condition in Patient 1. Patient 2 was able to flex the affected fingers slightly at discharge, although the movement was impossible to perform at admission. At 4 weeks after discharge, he was able to handle chopsticks and write letters holding the pen with the affected hand alone. The school teacher and piano instructor recognized the improvement in the movement of the affected hand following HFrTMS/OT.

Table 2. Changes in the measures applied for the study.

\begin{tabular}{|c|c|c|c|c|c|c|}
\hline & \multicolumn{3}{|c|}{ Patient 1} & \multicolumn{3}{|c|}{ Patient 2} \\
\hline & At admission & At discharge & $\begin{array}{l}4 \text { weeks after } \\
\text { discharge }\end{array}$ & At Admission & At discharge & $\begin{array}{c}4 \text { weeks after } \\
\text { discharge }\end{array}$ \\
\hline FMA (points) & 60 & 61 & 61 & 57 & 58 & 61 \\
\hline WMFT-FAS (points) & 59 & 61 & 61 & 69 & 71 & 72 \\
\hline STEF (points) & 45 & 48 & 55 & 85 & 90 & 97 \\
\hline Ten-second test (finger tapping) (times) & 12 & 11 & 16 & 10 & 13 & 17 \\
\hline
\end{tabular}


Table 3. Changes in ADL function using the affected upper limb.

\begin{tabular}{|c|c|c|}
\hline \multirow{3}{*}{ Patient 1} & At admission & $\begin{array}{l}\text { In need of some time to pass the affected arm through the sleeve of a shirt and fasten the buttons } \\
\text { on a shirt; Unable to grasp a bowl stably and throw the ball straight }\end{array}$ \\
\hline & At discharge & $\begin{array}{l}\text { Able to pass the affected arm through the sleeve of a shirt and fasten the buttons on a shirt more } \\
\text { smoothly; Able to grasp a bowl stably, and move the bowl to mouth smoothly; Able to throw a } \\
\text { small ball straight }\end{array}$ \\
\hline & 4 weeks after discharge & $\begin{array}{l}\text { Marked improvement in endurance during grasping a bowl; Able to throw a small ball more } \\
\text { powerfully than at admission }\end{array}$ \\
\hline \multirow{3}{*}{ Patient 2} & At admission & $\begin{array}{l}\text { Presence of enhanced spasticity, which prevented flexion of the affected fingers during motion; } \\
\text { In need of assistance device to handle chopsticks in affected hand; Need to place the unaffected } \\
\text { hand on the affected hand to write using a pen }\end{array}$ \\
\hline & At discharge & $\begin{array}{l}\text { Ability to flex the affected fingers slightly; Makes marked effort to eat food with the affected hand } \\
\text { alone }\end{array}$ \\
\hline & 4 weeks after discharge & $\begin{array}{l}\text { Able to use the affected hand more frequently in ADL; Able to handle chopsticks with the affected } \\
\text { hand alone; Able to write letters holding the pen with the affected hand alone; Able to write better } \\
\text { (assessed by the school teacher) and move affected hand and fingers more smoothly (assessed by } \\
\text { the piano instructor) }\end{array}$ \\
\hline
\end{tabular}

\section{Discussion}

This is the first clinical report of application of HF-rTMS over the lesional hemisphere combined with intensive OT for pediatric patients with upper limb hemiparesis. The results showed that the intervention was safe and feasible in two pediatric stroke patients. Our proposed protocol improved motor function of the affected upper limbs of these two children. In addition, the improvement of motor function of the affected upper limb was persistently seen up to 4 weeks after discharge.

So far, only a few researchers have introduced rTMS as a therapeutic tool for pediatric patients. Kirton et al. [9] applied LF-rTMS to the non-lesional hemisphere in pediatric post-stroke patients with upper limb hemiparesis aged between 8 and 20 years. They found that LF-rTMS over the non-lesional hemisphere was safe and improved motor function of the affected limbs. With regard to the application of HF-rTMS, Valle et al. [15] showed that application of HF-rTMS of 5-Hz over the lesional hemisphere in pediatric patients with cerebral palsy was safe and reduced the spasticity of the affected upper limb. However, there is no information on HF-rTMS applied over the lesional hemisphere in pediatric stroke patients. Although Rossi et al. reported that epileptic seizure, the most severe adverse effects of rTMS application, has been reported to occur with greater frequency in HF-rTMS protocol than low-frequency rTMS protocol, the two patients in our study completed the protocol without any adverse effects [16]. Therefore, it is considered that HFrTMS can be repeatedly applied over the lesional hemisphere in pediatric patients, if the patient meets the inclusion criteria for safety.

After the onset of stroke, adult stroke patients show increased inhibitory drive from the non-lesional hemisphere to the lesional hemisphere through corpus callo- sum and this increase of inhibition adversely influences motor functional recovery [17]. Accordingly, the application of suppressive LF-rTMS to the non-lesional hemisphere significantly improved motor function of the affected upper limb in adult post-stroke patients, through a reduction of interhemispheric inhibition towards the lesional hemisphere $[18,19]$. However, it has been reported that interhemispheric neural connections through the corpus callosum are immature in children under at least the age of 5 years [20]. This finding suggests that no pathological imbalance of hemispheric neural activity develops after the onset of stroke in pediatric patients as seen in adult patients. Furthermore, it has been reported that ipsilateral un-crossed corticospinal connections are identified in about two-thirds of children under the age of 10 years [21]. It seems likely that the non-lesional hemisphere could dominantly contribute to motor functional recovery in pediatric stroke patients with hemiparesis [21, 22]. Based on the above features in pediatric patients, we applied in this study excitatory HF-rTMS of $10-\mathrm{Hz}$ over the lesional hemisphere. The results showed improvement of motor function in the affected upper limb in both patients. Based on this result, one can speculate that our protocol of HF-rTMS over the lesional hemisphere resulted in functional neural reorganization in the lesioned hemisphere. In this regard, Johnston et al. [23] demonstrated that hemiparesis after stroke in pediatric patients shows better functional recovery than in adult patients and commented that this difference is due to the marked degree of neuroplasticity in pediatric patients. It is possible that repeated application of this 15-day protocol with an interval of several weeks or months could bring about further functional improvement.

We reported previously in adult stroke patients free of cognitive impairment the application of intensive OT program, which consisted of 60-min one-to-one training 
and 60-min self-exercise, together with LF-rTMS applied over the non-lesional hemisphere [8]. All participating adult patients completed the program without any difficulty. However, we expected that it would be difficult for pediatric patients to complete the program for lack of stamina and difficulty in persistent concentration on the task. Indeed, Brady et al. [24] have already suggested that the original protocol of constraint-induced movement therapy (CIMT) was troublesome and not accepted by pediatric patients. Therefore, we modified the intensive OT protocol for pediatric patients by shortening the training time, providing therapy in the home environment, and partly camouflaged the therapy within the context of play. Based on this concept, we reduced the duration of training, kept the parents close by and incorporated game elements and attractive objects like animals and painting into the training tasks. With these programs, the pediatric patients were able to concentrate in the 30-min one-toone training and 30-min self-exercise. Thus, it seems that these modifications enabled the participating children to concentrate on the tasks of the intensive OT program throughout 15-day hospitalization.

Importantly, the improved motor function was persistently seen until four weeks after discharge in both patients. In addition, both patients showed some score increase and improvement of ADL function after discharge. While the exact reason for these improvements was not investigated in this study, we speculate that continuous daily use of the affected upper limb after discharge facilitated the beneficial changes in the lesional hemisphere. During the period between discharge and four weeks later, motor functional improvement was more significant in Patient 2 than in Patient 1. This difference may partly depend on whether the affected side matches the side of handedness. Thus, this match in Patient 2 led to a more common use of the affected upper limb, which may have contributed to the better functional recovery of the affected upper limb compared to Patient 1.

The present study has certain limitations. First, this is a pilot study of only two subjects. The efficacy should be confirmed in a larger number of patients with a control group. Second, there are few standardized assessment scales of upper limb motor function in pediatric stroke patients. In many similar studies, assessment scales for adult patients have been modified and adapted for pediatric patients [25]. An appropriate scale of upper limb motor function that can be applied for pediatric stroke patients needs to be devised. Third, neither neuroimaging nor neurophysiological study was performed to evaluate functional changes in the brain in this study. Further studies using functional magnetic resonance imaging (fMRI) and TMS mapping techniques should be performed to examine the functional reorganization in the brain before and after the protocol.
In conclusion, the combination of HF-rTMS and intensive OT was safely completed and resulted in improvement of motor function of the affected upper limb in two children with hemiparesis. This protocol seems a suitable therapeutic intervention for this patient population, although its efficacy should be confirmed in a larger study.

\section{Acknowledgements}

The authors gratefully acknowledge the support and the participation of the patients in the study.

\section{REFERENCES}

[1] N. Agrawal, S. C. Johnston, Y. W. Wu, S. Sidney and H. J. Fullerton, "Imaging Data Reveal a Higher Pediatric Stroke Incidence than Prior US Estimates," Stroke, Vol. 40, No. 11, 2009, pp. 3415-3421. doi:10.1161/STROKEAHA.109.564633

[2] V. Ganesan, M. Prengler, M. A. McShane, A. M. Wade and F. J. Kirkham, "Investigation of Risk Factors in Children with Arterial Ischemic Stroke,” Annals of Neurology, Vol. 53, No. 2, 2003, pp. 167-173. doi:10.1002/ana.10423

[3] M. F. Rafay, A. M. Pontigon, J. Chiang, M. Adams, D. A. Jarvis, F. Silver, D. Macgregor and G. A. Deveber, "Delay to Diagnosis in Acute Pediatric Arterial Ischemic Stroke,” Stroke, Vol. 40, No. 1, 2009, pp. 58-64. doi:10.1161/STROKEAHA.108.519066

[4] J. A. Zimmer, B. P. Garg, L. S. Williams and M. R. Golomb, "Age-Related Variation in Presenting Signs of Childhood Arterial Ischemic Stroke,” Pediatric Neurology, Vol. 37, No. 3, 2007, pp. 171-175. doi:10.1016/j.pediatrneurol.2007.05.010

[5] M. Steinlin, I. Pfister, J. Pavlovic, R. Everts, E. Boltshauser, A. Capone Mori, D. Gubser Mercati, C. A. Hanggeli, E. Keller, J. Luetschg, J. Marcoz, G. P. Ramelli, E. Roulet Perez, T. Schmitt-Mechelke, M. Weissert and Swiss Societies of Paediatric Neurology and Neonatology, "The First Three Years of the Swiss Neuropaediatric Stroke Registry (SNPSR): A Population-Based Study of Incidence, Symptoms and Risk Factors," Neuropediatrics, Vol. 36, No. 2, 2005, pp. 90-97. doi:10.1055/s-2005-837658

[6] V. Ganesan, A. Hogan, N. Shack, A. Gordon, E. Isaacs and F. J. Kirkham, "Outcome after Ischaemic Stroke in Childhoo d," Developmental Medicine \& Child Neurology, Vol. 42, No. 7, 2000, pp. 455-461. doi:10.1017/S0012162200000852

[7] B. B Johansson, "Current Trends in Stroke Rehabilitation. A Review with Focus on Brain Plasticity," Acta Neurologica Scandinavica, Vol. 123, No. 3, 2011, pp. 147-159. doi:10.1111/j.1600-0404.2010.01417.x

[8] W. Kakuda, M. Abo, K. Kobayashi, R. Momosaki, A Yokoi, A. Fukuda, A. Ishikawa, H. Ito and A. Tominaga, "Low-Frequency Repetitive Transcranial Magnetic Stimulation and Intensive Occupational Therapy for Poststroke Patients with Upper Limb Hemiparesis: Preliminary Study of a 15-Day Protocol,” International Journal of Rehabilitation Research, Vol. 33, No. 4, 2010, pp. 339345. doi:10.1097/MRR.0b013e32833cdf10 
[9] A. Kirton, R. Chen, S. Friefeld, C. Gunraj, A. M. Pontigon and G. Deveber, "Contralesional Repetitive Transcranial Magnetic Stimulation for Chronic Hemiparesis in Subcortical Paediatric Stroke: a Randomised Trial,” The Lancet Neurology, Vol. 7, No. 6, 2008, pp. 507-513. doi:10.1016/S1474-4422(08)70096-6

[10] E. M. Wassermann, "Risk and Safety of Repetitive Transcranial Magnetic Stimulation: Report and Suggested Guidelines from the International Workshop on the Safety of Repetitive Transcranial Magnetic Stimulation, June 5-7, 1996," Electroencephalography and Clinical Neurophysiology, Vol. 108, No. 1, 1998, pp. 1-16. doi:10.1016/S0168-5597(97)00096-8

[11] D. J. Gladstone, C. J. Danells and S. E. Black, “The FuglMeyer Assessment of Motor Recovery after Stroke: A Critical Review of its Measurement Properties," Neurorehabilitation and Neural Repair, Vol. 16, No. 3, 2002, pp. 232-240. doi:10.1177/154596802401105171

[12] D. M. Morris, G. Uswatte, J. E. Crago, E. W. Cook and E. Taub, "The Reliability of the Wolf Motor Function Test for Assessing Upper Extremity Function after Stroke," Archives of Physical Medicine and Rehabilitation, Vol. 82, No. 6, 2002, pp. 750-755. doi:10.1053/apmr.2001.23183

[13] T. Kaneko and T. Muraki, "Development and Standardization of the Hand Function Test," Bulletin of Allied Medical Sciences Kobe, Vol. 6, 1990, pp. 49-54.

[14] T. Hatanaka, T. Koyama, M. Kanematsu, N. Takahashi, K. Matsumoto and K. Domen, “A New Evaluation Method for Upper Extremity Dexterity of Patients with Hemi- paresis after Stroke: The 10-Second Tests,” International Journal of Rehabilitation Research, Vol. 30, No. 3, 2007, pp. 243-247. doi:10.1097/MRR.0b013e3282ab961d

[15] A. C. Valle, K. Dionisio, N. B. Pitskel, A. Pascual-Leone, F. Orsati, M. J. Ferreira, P. S. Boggio, M. C. Lima, S. P. Rigonatti and F. Fregni, "Low and High Frequency Repetitive Transcranial Magnetic Stimulation for the Treatment of Spasticity," Developmental Medicine \& Child Neurology, Vol. 49, No. 7, 2007, pp. 534-538. doi:10.1111/j.1469-8749.2007.00534.X

[16] S. Rossi, M. Hallett, P. M. Rossini, A. Pascual-Leone and Safety of TMS Consensus Group, "Safety, Ethical Considerations and Application Guidelines for the Use of Transcranial Magnetic Stimulation in Clinical Practice and Research," Clinical Neurophysiology, Vol. 120, No. 12, 2009, pp. 2008-2039. doi:10.1016/j.clinph.2009.08.016
[17] N. Murase, J. Duque, R. Mazzocchio and L. G. Cohen, “Influence of Interhemispheric Interactions on Motor Function in Chronic Stroke," Annals of Neurology, Vol. 55, No. 3, 2004, pp. 400-409. doi:10.1002/ana.10848

[18] N. Takeuchi, T. Chuma, Y. Matsuo, I. Watanabe and K. Ikoma, "Repetitive Transcranial Magnetic Stimulation of Contralesional Primary Motor Cortex Improves Hand Function after Stroke," Stroke, Vol. 36, No. 12, 2005, pp. 2681-2686. doi:10.1161/01.STR.0000189658.51972.34

[19] F. Fregni, P. S. Boggio, A. C. Valle, R. R. Rocha, J. Duarte, M. J. Ferreira, T. Wagner, S. Fecteau, S. P. Rigonatti, M. Riberto, S. D. Freedman and A. Pascual-Leone, “A Sham-Controlled Trial of a 5-Day Course of Repetitive Transcranial Magnetic Stimulation of the Unaffected Hemisphere in Stroke Patients,” Stroke, Vol. 37, No. 8, 2006, pp. 2115-2122. doi:10.1161/01.STR.0000231390.58967.6b

[20] F. Heinen, F. X. Glocker, U. Fietzek, B. U. Meyer, C. H. Lucking and R. Korinthenberg, “Absence of Transcallosal Inhibition Following Focal Magnetic Stimulation in Preschool Children,” Annals of Neurology, Vol. 43, No. 5, 1998, pp. 608-612. doi:10.1002/ana.410430508

[21] K. Muller, F. Kass-Iliyya and M. Reitz, “Ontogeny of Ipsilateral Corticospinal Projections: A Developmental Study with Transcranial Magnetic Stimulation,” Annals of Neurology, Vol. 42, No. 5, 1997, pp. 705-711. doi:10.1002/ana.410420506

[22] R. Benecke, B. U. Meyer and H. J. Freund, "Reorganisation of Descending Motor Pathways in Patients after Hemispherectomy and Severe Hemispheric Lesions Demonstrated by Magnetic Brain Stimulation,” Experimental Brain Research, Vol. 83, No. 2, 1991, pp. 419-426. doi:10.1007/BF00231167

[23] M. V. Johnston, "Plasticity in the Developing Brain: Implications for Rehabilitation,” Developmental Disabilities Research Reviews, Vol. 15, No. 2, 2009, pp. 94-101. doi:10.1002/ddrr.64

[24] K. Brady and T. Garcia, "Constraint-Induced Movement Therapy (CIMT): Pediatric Applications,” Developmental Disabilities Research Reviews, Vol. 15, No. 2, 2009, pp. 102-111. doi:10.1002/ddrr.59

[25] A. Gordon, A. Connelly, B. Neville, F. Vargha-Khadem, N. Jessop, T. Murphy and V. Ganesan, "Modified Constraint-Induced Movement Therapy after Childhood Stroke,” Developmental Medicine \& Child Neurology, Vol. 49, No. 1, 2007, pp. 23-27. doi:10.1017/S0012162207000072.x 